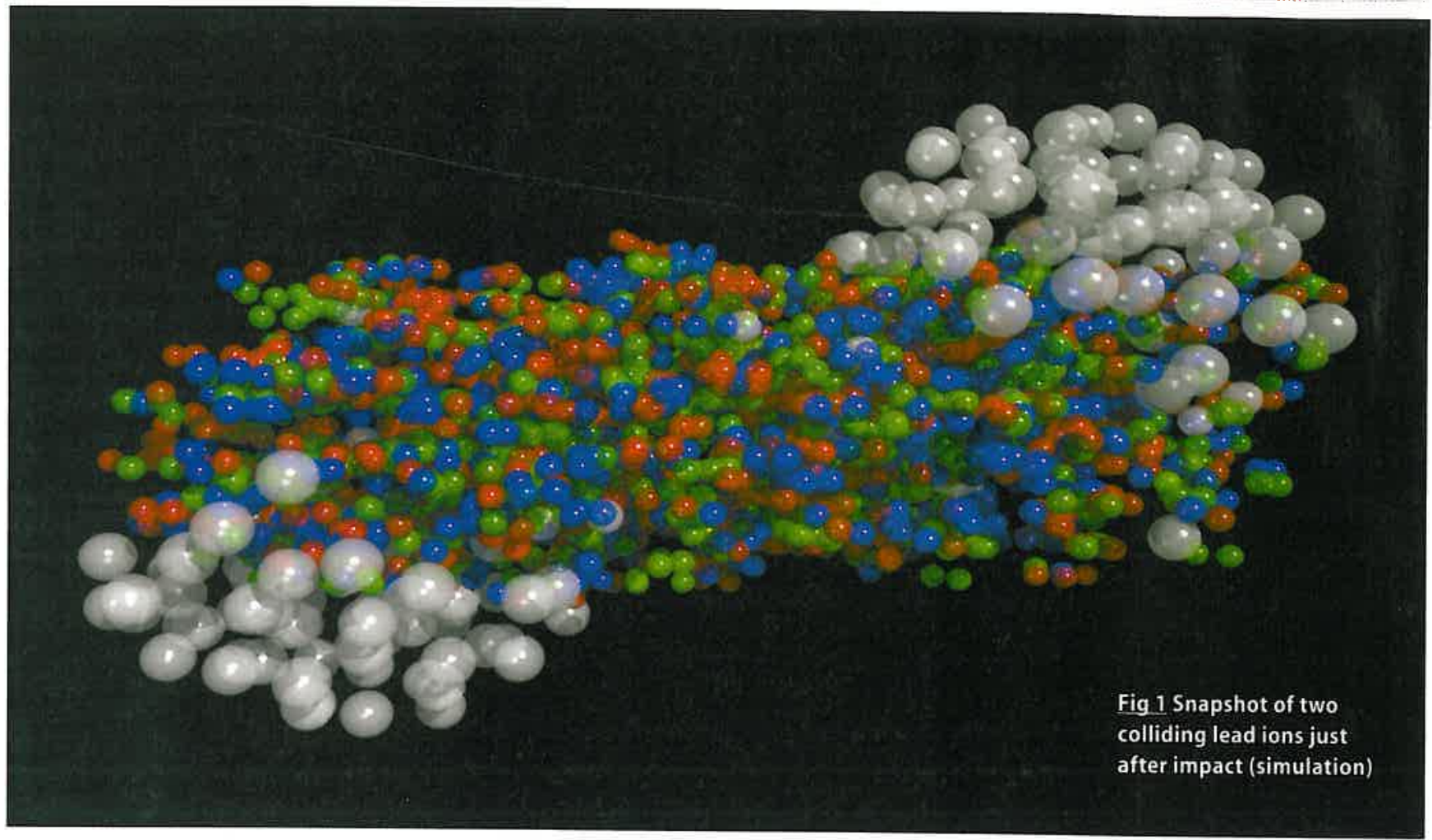

\title{
First data on the quark-gluon plasma reported at CERN
}

$\mathrm{O}$ ur universe is expanding from an initial phase of near infinite energy density and temperature. The only "data" we have about the very beginning - the Big Bang - is the very existence of the expanding universe. A subsequent phase which begins before the femtosecond era and is believed to have prevailed until about 10 microseconds was equally hypothetical up to now: The quark-gluon plasma phase represents a state of quasi-free, deconfined quarks - the most primary form of matter governed by the strong force. The fundamental theory of the strong interaction, Quantum Chromodynamics (QCD), that predicts 'asymptotic freedom' at very high temperature, leads us to expect such a state of deconfined quarks. This means a drastic reduction of the extremely strong attraction mediated by gluon exchange in low temperature states. With "low" we still mean about $2.10^{12}$ Kelvin, at which point the thermal wavelength is about equal to the mesonic sizes of about 0.5 fermi. Freedom and, therefore, quark deconfinement is thus expected to set in once the energy density steps well above this limit: confined quarks in protons, neutrons, mesons give way to a colour-conducting quark-gluon plasma. A more detailed understanding of the cosmological plasma phase would require data detailing its moment of creation from a preceding "Grand Unification" era (which is supposed to consist of super-energetic $\mathrm{X}$-particles experiencing the electromagnetic and weak interactions in as strong a manner as the strong force itself), as well as defining the point at which the plasma freezes out into confined protons and neutrons. The equation of state - the relation between pressure and energy density would also be important for the detailed cosmological dynamics. QCD makes only semi-qualitative predictions here (confinement - deconfinement belongs to the less well established non-perturbative sectors of QCD).

CERN, the European laboratory for particle physics in Geneva, has just made public the results of a five-year program of heavy-ion collision experiments. These data provided the first information on the existence of a new deconfined phase of matter, and on the energy density and temperature that characterise the phase transition point from deconfined plasma to confined hadrons. According to the results, which confirm the estimates provided by QCD lattice theory, the phase transition takes place at a temperature $\mathrm{T}=175 \mathrm{MeV}$ and at an energy density of about $1 \mathrm{GeV}$ per cubic fermi. A "classical" translation of these conditions results in an estimate of the pressure corresponding to the hadronization point of about $10^{32}$ $\mathrm{kg} / \mathrm{cm}^{2}$ (ie. about 50 solar masses per $\mathrm{cm}^{2}$ ). Thus the emerging experimental data has pushed cosmology an entire era backwards towards the beginning and given the fundamental QCD theory new momentum in its non-perturbative sector.

Seven experiments at the CERN SPS 33 TeV Pb beam facilities (NA44/45/49/50/52 and WA 97/98) have contributed complementary aspects of those overall findings, which were presented in a public seminar and press conference held at CERN on February 10. The CERN program will continue this year for finer details. The physicists are also engaged in preparation of a new round of quark-gluon plasma studies in collider experiments beginning at the Brookhaven National Laboratory relativistic heavy-ion collider (RHIC) later this year, and at the Large Hadron Collider (LHC) of CERN in 2005. At the 10-to 300-fold higher centre-of-mass energies that will be provided here, intricate details of the equation of state of QCD matter are expected to unfold. 